\title{
Experimental study of wave propagation in heterogeneous materials
}

\author{
M. Donà and M. Lombardo and G. Barone \\ School of Civil and Building Engineering, Loughborough University, UK \\ 15th The Fifteenth International Conference on Civil, Structural and \\ Environmental Engineering Computing, CC 2015; \\ Prague; Czech Republic; 1-4 September 2015
}

\begin{abstract}
The phenomenon of wave propagation through concrete materials is affected by dispersion due to its intrinsic heterogeneous microstructure. Previous experiments have shown an increase of phase velocity at high frequencies. This behaviour cannot be analytically described by the classical elasticity theory, due to its non-dispersive nature. Instead, enhanced theories can be adopted. In this work the dynamically consistent non local model, able to take into account the microstructural effects by two additional length scale parameters, is retrieved. The main subject of this contribution is the experimental identification of the dispersive behaviour of cementitious materials and the validation of the gradient continuum to predict the dispersion of the wave born out of the heterogeneity of the material. The proposed work extends the applicability of non-local theories from a purely theoretical/analytical domain to the laboratory territory.
\end{abstract}

Keywords: Wave propagation, dispersion, gradient-enriched continuum.

\section{Introduction}

The propagation of elastic waves is largely used in many inspection techniques to estimate the quality and the integrity of a material. For example, concrete strength and Young's modulus are related through empirical relations to the pulse velocity, measured as the ratio between the travelled distance and the arrival time of the waveform, identified as the first disturbance detected (BS EN 12504-4:2004 (2004); ASTM C215-14 (2014)). Further, knowledge of how stress waves propagate through a medium is important for non-destructive testing techniques based on ultrasonic testing and acoustic emissions.

Concrete is a composite material where coarse ( 3 to $30 \mathrm{~mm}$ ) and fine ( 0.1 to $3 \mathrm{~mm}$ ) 
aggregates are joined together by a cement paste, resulting in a random heterogeneous microstructure (Aggelis and Polyzos (2004)). When elastic waves propagate through such material, the internal inhomogeneities cause the generated signal to travel at different velocities and to follow different travel paths before arriving to the receiver, exhibiting a dispersive behaviour, where waves of different frequencies travel at different speeds. In particular, this behaviour is more noticeable when the wavelengths are comparable or smaller than the size of the microstructure (the aggregate size in this particular case).

The ultrasonic pulse velocity (UPV) technique is one of the most popular method used for the assessment of structural integrity. For accurate characterisation of concrete elements, since different variables affect the pulse velocity measurement, the wave characteristics should be considered to complement the wave velocity information. In fact, within the frequency range $10-300 \mathrm{kHz}$, the wave velocity increases with higher frequencies (Popovics et al. (1990); Aggelis et al. (2004)). This increment is affected by several factors like moisture content, temperature, path length, shape and size of the specimen, cracks and voids. (BS EN 12504-4:2004 (2004); Malhotra and Carino (2004)). The effect of aggregate size has been object of previous research as for example in (Jacobs and Owino (2000); Molero et al. (2011)) who studied the attenuation of Rayleigh waves in concrete materials using laser ultrasonics and its relation to scattering phenomena and absorption, showing as the latter rules the attenuation mechanisms while the aggregate size does not affect it. Attenuation and propagation of ultrasonic waves have been used to obtain information about the size distribution of the aggregate in concrete beams (Gaydecki et al. (1992)). It has been found that the amount and type of aggregate significantly increase the pulse velocity if compared with cement paste (Malhotra and Carino (2004); Popovics et al. (1990)). In order to specifically study the effects of the aggregate size on the wave propagation phenomena, a few researchers (Anugonda et al. (2001); Becker et al. (2003); Molero et al. (2011)) tested specimens made of Portland cement, water and glass spheres of a uniform diameter. Among other variables, it is worth mentioning that the pulse velocity is also affected by water-cement (w/c) ratio; increasing $\mathrm{w} / \mathrm{c}$ ratios correspond to reduced pulse velocities (Aggelis and Polyzos (2004); Aggelis et al. (2004)).

Reliable non-destructive testing therefore requires better interpretation of the experimental observations and the benefit of analytical predictions is that they facilitates increased understanding of nascent phenomena. Since in many practical situations the microstructural features significantly influence the global response, accurate modelling of wave propagation in heterogeneous media requires that the microstructure is accounted for. Discrepancies between experimental observations and theoretical predictions based on classical theories show that the latter are not adequate to capture wave dispersion because they do not accommodate information about the microstructure within the continuum model. Several other formulations have been proposed to fix this issue; analysis methods based on discrete modelling and on enhanced continuum theories are the most common technique. In the first case, the heterogeneities are modelled as a lattice of masses linked together through an energy balance; Iliopoulos et al. (2015), for example, used a lattice model where the aggregates are simulated 
as masses linked together through elastic springs representing the concrete matrix. Instead, in the enhanced continuum approaches the medium is treated as homogeneous equivalent to the heterogeneous one and the microstructure is taken into account by additional internal variables (Carta and Brun (2012)). Mindlin (Mindlin (1964)) has been one of the first to develop one of these models, followed by many others as (Chen and Fish (2001); Askes et al. (2007); Bennett et al. (2007a); Askes and Aifantis (2011)) where the classical continuum theory is enriched with higher order gradients of the field variables accompanied by length scale parameters.

Many studies available in literature propose new analytical models based on enriched theories but only limited research has been devoted to the experimental identification of the additional characteristic length scale parameters from wave propagation measurements (Carta et al. (2012)); this paper represents a step forward in this direction. It focuses on the dynamically consistent gradient elasticity model (Metrikine and Askes (2002)) where two additional terms related to stiffness and inertia contribution are introduced. It has been chosen because in its simplicity guarantees physical consistency and numerical stability. If compared with other methods, it allows the representation of the monotonically increasing dispersive behaviour of concrete which is in good agreement with experimental evidence.

In the proposed work, elastic longitudinal waves have been applied on one end of a concrete beam and recorded on the opposite one using piezoelectric transducers. In order to obtain measurements at several distances, the concrete beam has been cut into shorter beams and the experiment repeated for each part. In particular, in this contribution, the dispersion behaviour of concrete is experimentally investigated and compared with the analytical prediction with the aim to demonstrate the potential and limitations of this type of gradient model, but also to introduce the work that will be accomplished in future investigations.

This paper is structured in five sections. First, the dynamically consistent gradient elasticity model is reviewed and its properties briefly discussed. Then, the propagation of longitudinal waves in concrete material is discussed in relation to the gradient elasticity model presented in the previous section. In Section 4 the experimental equipment and the testing procedure are described. Section 5 reviews the different methods used to obtain the group velocity and phase velocity. Finally the results of the tests on different concrete sample and, in particular, the dispersion curves are presented.

\section{Wave Dispersion Model}

Aim of this section is to investigate the suitability of the so called dynamically consistent gradient elasticity theory to predict wave dispersion in concrete (Metrikine and Askes (2002)). The derivation of the dispersion curve for a one-dimensional problem will be briefly recapped and comparison between analytical results and experimental observations will demonstrate that the elastic wave dispersion in concrete can be captured with an appropriate selection of the microstructural lengths. 


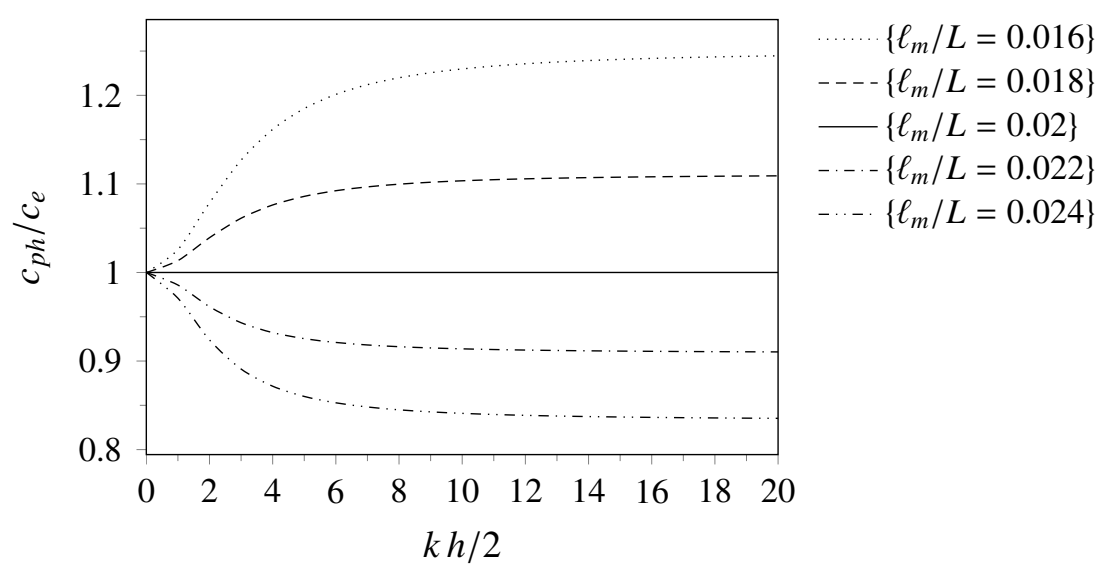

Figure 1: Dispersion curves for wave propagation in an axial bar with dynamically consistent gradient elasticity $\left(\ell_{s} / L=0.02\right)$

The differential equation of motion for the one-dimensional problem reads:

$$
\rho \ddot{u}-\rho \ell_{m}^{2} \ddot{u} "=E u^{\prime \prime}-E \ell_{s}^{2} u^{\prime \prime \prime \prime},
$$

where $u=u(x)$ is the axial displacement; $\rho$ is the mass density; $E$ is the Young's modulus; $\ell_{s}$ and $\ell_{m}$ are the length scale parameters related with the higher-order stiffness and inertia, respectively. In Eq. (1) the superimposed dot denotes time derivative, while the inverted comma / denotes spatial derivative.

Assuming $u$ as a general harmonic wave of the form $u=A \exp \left(i k\left(x-c_{p h} t\right)\right)$, the dispersion relation is obtained from Eq. (1) as follows:

$$
-\rho k^{2} c_{p h}^{2}-\rho \ell_{m}^{2} k^{4} c_{p h}^{2}=-E k^{2}-E \ell_{s}^{2} k^{4},
$$

where $A$ is the amplitude, $k$ is the wave number and $c_{p h}$ is the phase velocity. Eq. (2) can also be written in terms of the phase velocity and the wave number as:

$$
\frac{c_{p h}^{2}}{c_{e}^{2}}=\frac{1+\ell_{s}^{2} k^{2}}{1+\ell_{m}^{2} k^{2}}
$$

where the velocity of classical elasticity $c_{e}=\sqrt{E / \rho}$. Eq. (3) is the dispersion relation for the dynamically consistent model; depending on the values given to the length scale parameters, it predicts different material behaviours. Fig. 1 shows different dispersion curves for a beam of length $L=1 \mathrm{~m}$ and square cross section (side $h=0.1 \mathrm{~m}$ ). The properties assigned to the material, Young's modulus $E=30 \mathrm{GPa}$ and the density $\rho=2400 \mathrm{~kg} / \mathrm{m}^{2}$, are similar to concrete. Five combinations of $\left\{\ell_{s} ; \ell_{m}\right\}$ have been considered: $\ell_{s} / L=\{0.02\} ; \ell_{m} / L=\{0.016,0.018,0.02,0.022,0.024\}$. It can be observed that when the length scale parameter related with the micro-inertia $\ell_{m}$ is lower than $\ell_{s}$, the phase velocity $c_{p h}$ is larger than the phase velocity of the classical elasticity $c_{e}$, while the opposite is valid when $\ell_{s}<\ell_{m}$. It is worth mentioning here that when $\ell_{s}=\ell_{m}$, the classical elasticity model with no dispersive behaviour is restored (continuous line in Fig. 1). 


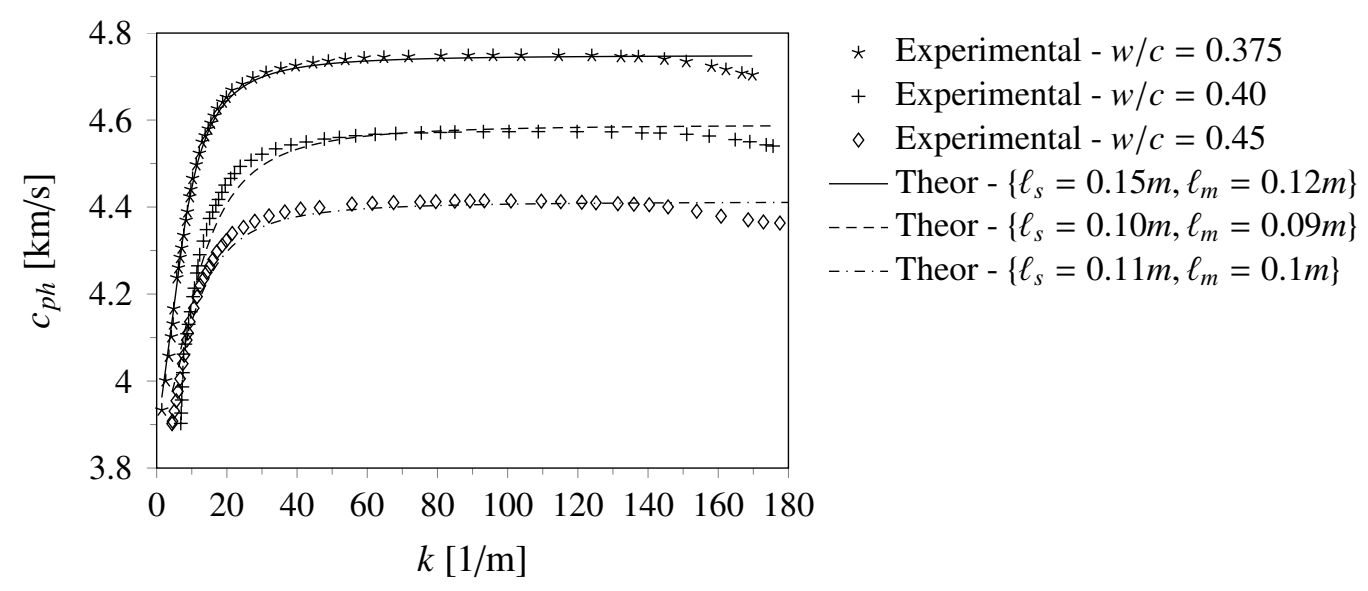

Figure 2: Experimental dispersion curves for wave propagation in concrete for the same aggregate-cement ratio $(\mathrm{a} / \mathrm{c}=3)$ and four different water-cement ratio $(\mathrm{w} / \mathrm{c})$ (Philippidis and Aggelis (2005)) and corresponding dispersion curve obtained with the dynamically consistent model.

The dynamically consistent theory has been proposed to model wave dispersion in discrete lattice (Askes et al. (2007)) or one-dimensional laminate (Bennett et al. (2007b)) where higher wave numbers propagate slower than the lower wave numbers, that is when $\ell_{s}<\ell_{m}$. Instead, it has been experimentally proved that concrete exhibits the opposite behaviour. As it can be observed in Fig. 2, the experimental dispersion results are in good agreement with the analytical curves obtained from the dynamically consistent model when the microstiffness length scale $\ell_{s}$ is higher than the microinertia contribution $\ell_{m}$.

\section{Materials and equipments}

The aim of this section is to present the preliminary experimental investigation that has been carried out in concrete samples in order to optimize the procedure of pulse velocity measurements at different frequencies. The experiments involved two different mixes, the first one made of sand while the second one of sand and aggregate with average size of $12 \mathrm{~mm}$, both mixed together with Portland cement and water (water over cement ratio w/c of 0.4). The concrete beam samples have a square section, with side of $100 \mathrm{~mm}$, and length ranging from $100 \mathrm{~mm}$ to $1 \mathrm{~m}$. These values have been chosen following the Standard (BS EN 12504-4:2004 (2004)) which suggests a minimum length of $100 \mathrm{~mm}$ to avoid local effects of the aggregate, and a minimum size of the specimen related to the wavelength of the transducer. In this case the minimum recommended lateral dimension was $74 \mathrm{~mm}$ for the $54 \mathrm{kHz}$ transducer. Considering the significant effect of the moisture content on the pulse velocity, the same curing conditions on the hydration of the cement has been adopted for the different specimens. Figure 3 shows the cross sections for the two investigated samples: Fig. 3a shows the 


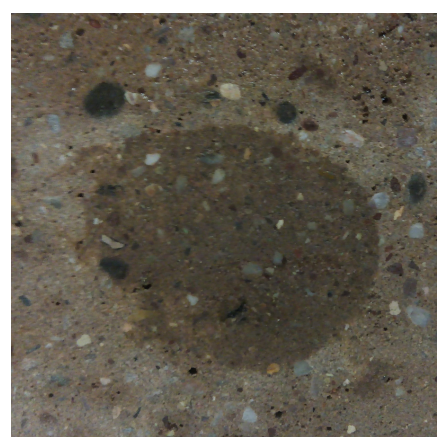

(a) Mortar



(b) Max aggr. size $=12 \mathrm{~mm}$

Figure 3: Cross sections of the tested concrete beams

beam made of mortar only; Figs. 3b shows the beam's section made of aggregate with maximum size of $12 \mathrm{~mm}$. In the last case the amount of aggregate and the amount of sand were $80 \%$ and $40 \%$ of the total volume, respectively.

The experimental arrangement consists of two piezoelectric transducers coupled to both end faces of the concrete beams using medium coupling grease (see Fig. 4). One of the transducer was excited to resonance by a Pundit pulser unit through a voltage pulse excitation while the second transducer was acting as receiver. The pulse applied to excite the transducer had width on the range $1 \mu \mathrm{s}$ to $10 \mu \mathrm{s}$ and the voltage between $125 \mathrm{~V}$ to $500 \mathrm{~V}$. Even though the ultrasonic pulser is mainly used for field inspections, the repeatability of the generated waves allows to perform the experiment on different beams assuming that the same wave is applied each time. Similar experimental set-up has been already used by several researchers for the laboratory experiments (Popovics et al. (1990); Long (2000)).

In order to study a range of frequencies where the material exhibits dispersive behaviour, three pairs of narrow-band resonant transducers with different centre frequency $(54 \mathrm{kHz}, 150 \mathrm{kHz}$ and $250 \mathrm{kHz})$ have been used, covering then the lower end of the ultrasonic range. For each sample, the test has been repeated several times, showing good agreement on the measured shape and arrival time of the pulse wave.

\subsection{The phase spectrum method - Fourier transform}

Several techniques are available to evaluate the phase velocity from wave signals, as for example the amplitude spectrum method, where the phase velocity is calculated using the longitudinal resonant frequencies combined with the mode numbers and the distance between the boundaries of the specimen (Pialucha et al. (1989)); or the $\pi$-point phase method which consists of determining the half-wavelength of an harmonic wave by varying the distance between transmitter and receiver transducers and determining the shift required to change the phase of the received signal by $\pi$; the phase velocity is then computed from continuous monochromatic waves or narrow band pulses (Papadakis (1976)). In this work the phase spectrum approach, as de- 


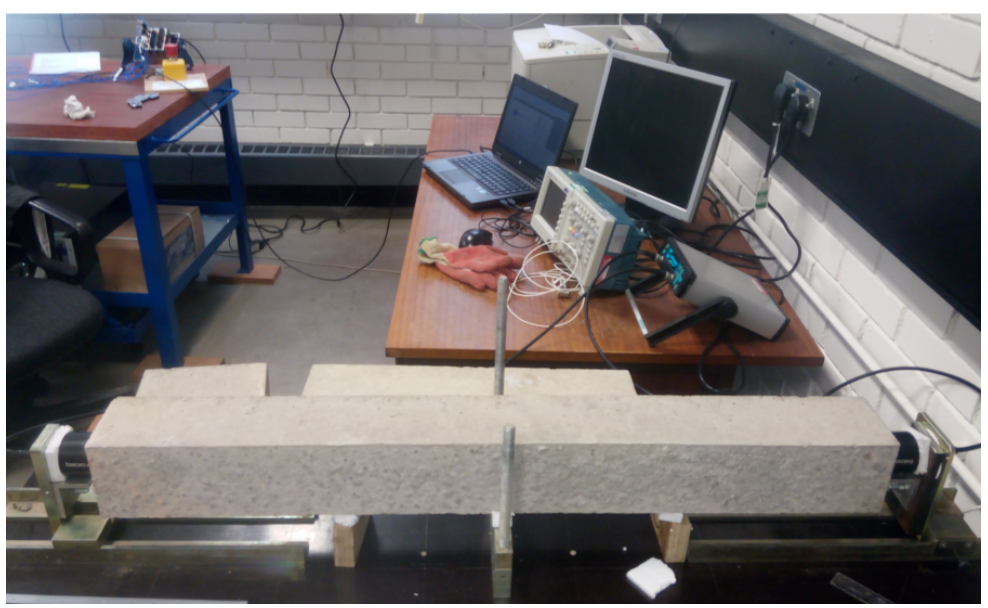

Figure 4: Experimental set-up

scribed by Sachse and Pao (1978), is adopted to determine the dispersion relation of the phase velocity $c_{p h}(\omega)$. Given the two signals, $y_{1}(t)$ and $y_{2}(t)$, generated by the same source type and occurred at different points, the technique consists in first computing the Fourier transform of the signals, and then unwrapping the phase spectra $\phi_{p h}(\omega)$ to obtain a continuous function. The phase velocity $c_{p h}(\omega)$ is finally evaluated as the difference between the wave angular frequency $\omega$ times the distance between the path length $z$, and the difference in phase between the two unwrapped phase spectra $\phi_{p h_{1}}(\omega)$ and $\phi_{p h_{2}}(\omega)$, as follow:

$$
c_{p h}(\omega)=\frac{z \omega}{\phi_{p h_{1}}(\omega)-\phi_{p h_{2}}(\omega)} .
$$

This method requires two signals with compact support (decay rapidly) to avoid leakage of frequency components; furthermore only one wave packet should be present in the signal to avoid overlapping. Particular attention has to be provided in the unwrapping procedure as an error in the continuous function can affects all the subsequent phase angle values at higher frequencies and as consequence the phase velocities (Long (2000)).

\section{Results and discussion}

In this section, the main experimental results are presented and discussed using different plots. In the following, the amplitude is normalised with respect to the maximum recorded value, based on the assumption that the wave velocity does not depend on the amplitude of the signal. Fig 5 shows the recorded normalised amplitude response (Ampl [\%]) for the mortar sample, measured with three types of transducers (resonant frequencies $54 \mathrm{kHz}, 150 \mathrm{kHz}$ and $250 \mathrm{kHz}$ ). Two different beams' lengths $L_{1}=195 \mathrm{~mm}$ and $L_{2}=300 \mathrm{~mm}$, corresponding to two signals $S_{1}$ and $S_{2}$, have been considered. In order to avoid overlaps, a $160 \mu \mathrm{s}$ signal has been used, i.e. shorter than three times its 

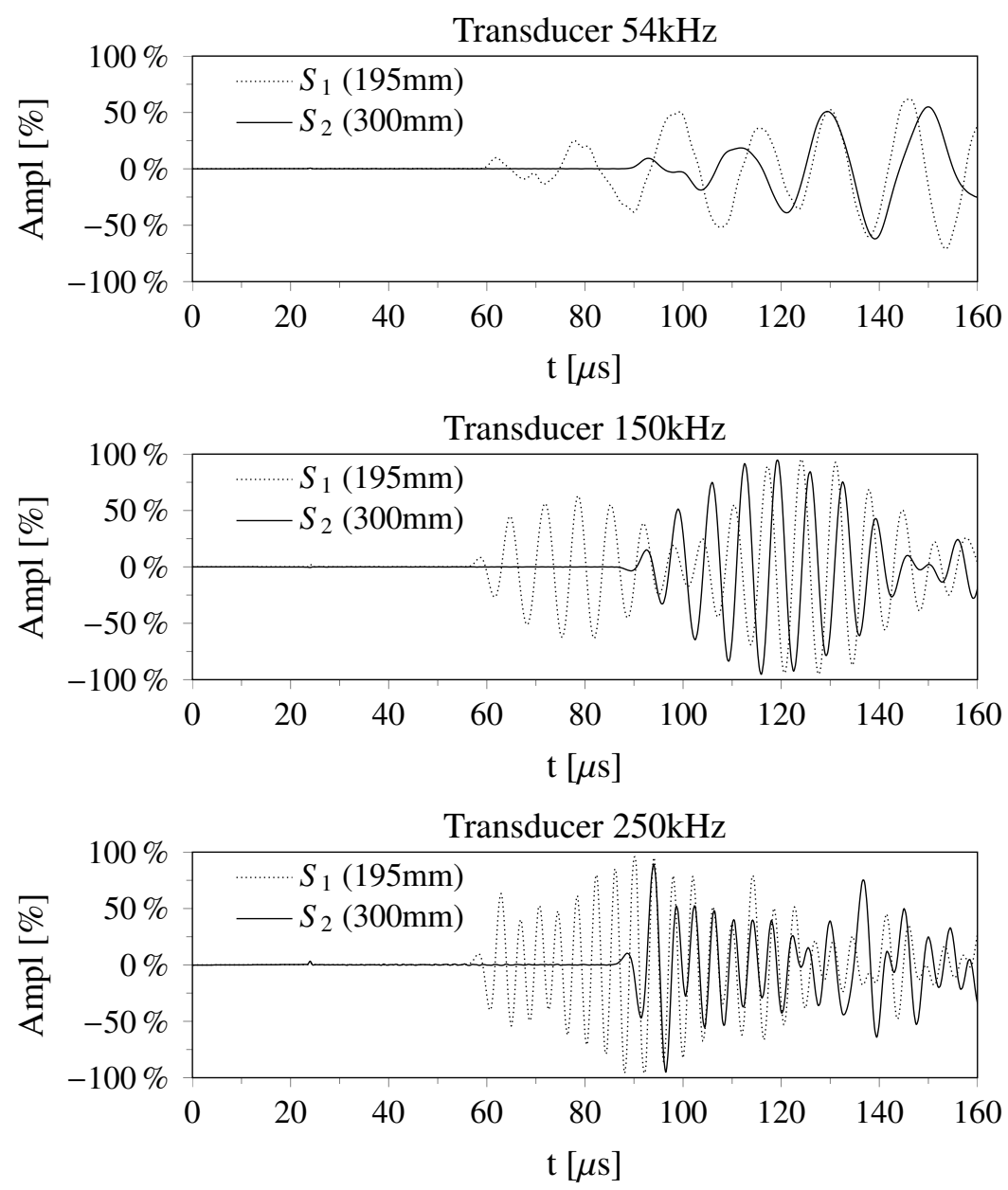

Figure 5: Mortar sample - Normalised amplitude response (Ampl [\%]) of two signals $S_{1}$ and $S_{2}$ corresponding to two different lengths of the beam $L_{1}=195 \mathrm{~mm}$ and $L_{2}=300 \mathrm{~mm}$, for three types of transducers (resonant frequency $54 \mathrm{kHz}, 150 \mathrm{kHz}$ and $250 \mathrm{kHz})$

arrival time ( $55 \mu \mathrm{s}$ for $\left.L_{1}=195 \mathrm{~mm}\right)$.

If we compare the shape of the received signal, for the same couple of transducers, we can observe that they look similar. The dominant frequency of the signal is the same as the resonant frequency of the transducer as we can clearly see in the amplitude spectra shown in Fig. 6 or Fig. 7 for the transducers with resonant frequencies of $54 \mathrm{kHz}$ and $150 \mathrm{kHz}$, respectively.

As suggested by Philippidis and Aggelis (2005), in order to obtain a consistent dispersion curve, the phase spectrum method was performed windowing the signal to reduced portions. Fig. 6 shows the amplitude spectrum (Ampl [\%]) of signal $S_{1}$ recorded at the positions $L_{1}=195 \mathrm{~mm}$, with the transducer with resonant frequency of $54 \mathrm{kHz}$, as well as two portions of it, $S_{1,2 \mathrm{pks}}$ and $S_{1,4 \mathrm{pks}}$, corresponding to the signal truncated at the second and fourth peak, respectively. It can be observed that the original signal $S_{1}$ has high amplitude in a clear frequency range around the resonance 

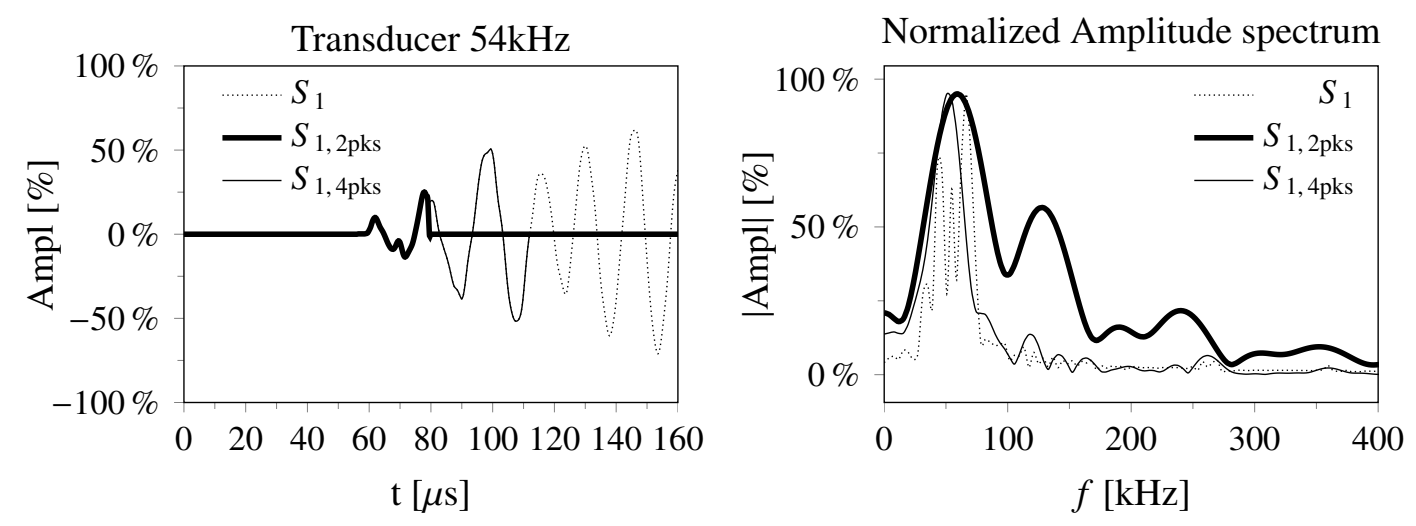

Figure 6: Mortar sample - Normalised amplitude response (Ampl [\%]) and amplitude spectrum (|Ampl|) of signal $S_{1}$ and two portions of it $S_{1,2 \mathrm{pks}}$ and $S_{1,4 \mathrm{pks}}$ recorded at the positions $L_{1}=195 \mathrm{~mm}$, for the transducer with resonant frequency of $54 \mathrm{kHz}$
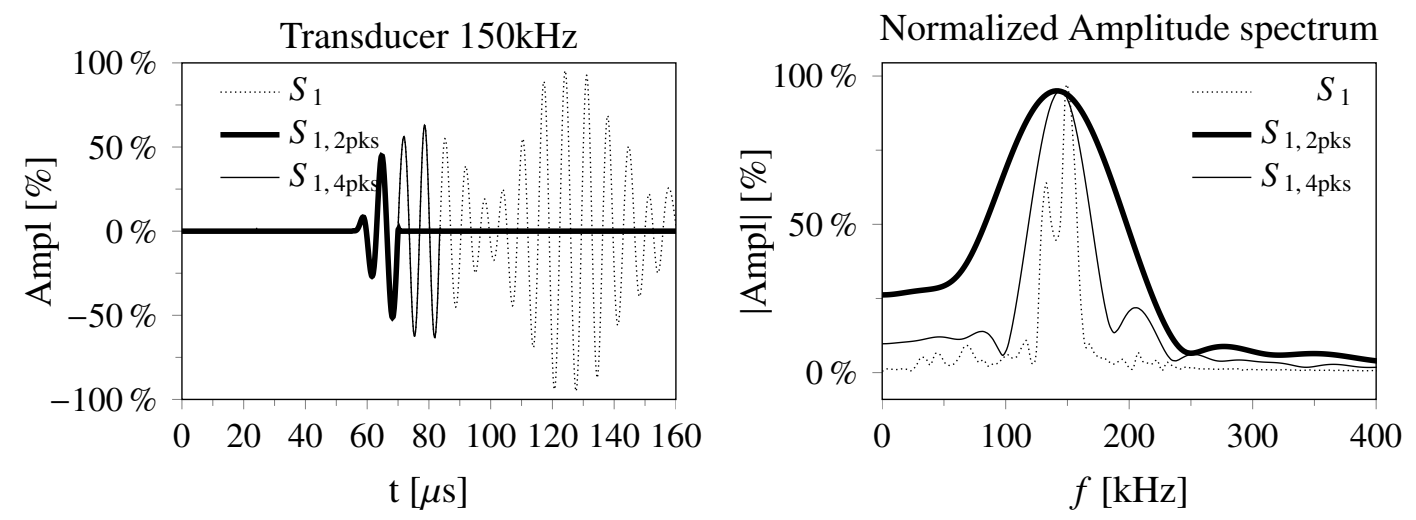

Figure 7: Mortar sample - Normalised amplitude response (Ampl [\%]) and amplitude spectrum (|Ampl|) of signal $S_{1}$ and two portions of it $S_{1,2 \mathrm{pks}}$ and $S_{1,4 \mathrm{pks}}$ recorded at the positions $L_{1}=195 \mathrm{~mm}$, for the transducer with resonant frequency of $150 \mathrm{kHz}$

of the transducer, as well as the $S_{1,4 \mathrm{pks}}$ while for the signal $S_{1,2 \mathrm{pks}}$, further relevant frequencies appears. This suggests that the truncated signals have to be carefully used in the phase spectrum method, where the phase of the signal plays a key role in the quantification of the phase velocity. Similarly, Fig. 7 shows the same for the transducer with resonant frequency of $150 \mathrm{kHz}$. Again, the less peaks we consider the smoother is the Fourier transform, but resulting to erroneously account for frequencies far from the resonance one.

The second example, shown in Fig. 8, is the signal recorded after propagating through the concrete samples of two different lengths $L_{1}=600 \mathrm{~mm}$ and $L_{2}=800 \mathrm{~mm}$, for the three types of transducers used in this work. In this case the time arrival for the shorter beam is about $150 \mu \mathrm{s}$, so the duration of the signal was chosen to be $400 \mu \mathrm{s}$. It can be observed that only for the $54 \mathrm{kHz}$ transducer the signals are smooth, while for the remaining two it is more irregular, especially for the length of $800 \mathrm{~mm}$. This dis- 



Figure 8: Concrete sample - Normalised amplitude response (Ampl [\%]) of two signals $S_{1}$ and $S_{2}$ corresponding to two different positions $L_{1}=600 \mathrm{~mm}$ and $L_{2}=$ $800 \mathrm{~mm}$ of the transducers, for three types of transducers (resonant frequency $54 \mathrm{kHz}$, $150 \mathrm{kHz}$ and $250 \mathrm{kHz})$

turbance can be explained as higher frequencies are strongly attenuated and the noise over signal ratio increases for increasing length of the beam sample.

Signals truncated at the first peak $S_{1,1 \mathrm{pk}}$ and third peak $S_{1,3 \mathrm{pks}}$ have been represented using the Fourier transform, for the concrete sample of $L_{1}=600 \mathrm{~mm}$ and transducer with resonant frequency of $54 \mathrm{kHz}$ (Fig. 9); while Fig. 10 shows the original signal $S_{1}$, the signal truncated at the second peak $S_{1,2 \mathrm{pk}}$ and the signal truncated at the fourth peak $S_{1,4 \mathrm{pk}}$ for the transducer with resonant frequency of $150 \mathrm{kHz}$. As for the mortar specimen, even though the portions of the signal have a smoother frequency spectrum, they include different frequency content.

An attempt to evaluate the phase velocity starting from the frequency spectrum of the original signal and the truncated versions has been conducted using the phase spectrum method presented in section 3.1. The resulting dispersion curves for three different portions of the signal are shown for the mortar in Fig. 11 and for the concrete 

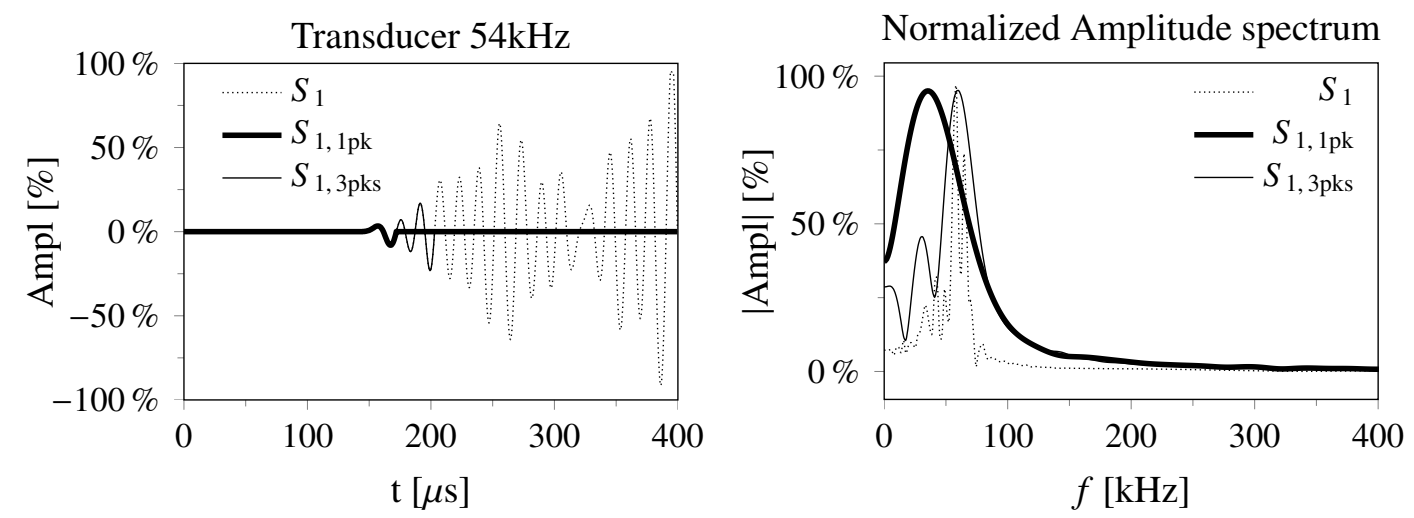

Figure 9: Concrete sample - Normalised amplitude response (Ampl [\%]) and amplitude spectrum (|Ampl|) of signal $S_{1}$ and two portions of it $S_{1,1 \mathrm{pk}}$ and $S_{1,3 \mathrm{pks}}$ recorded at the positions $L_{1}=600 \mathrm{~mm}$, for the transducer with resonant frequency of $54 \mathrm{kHz}$
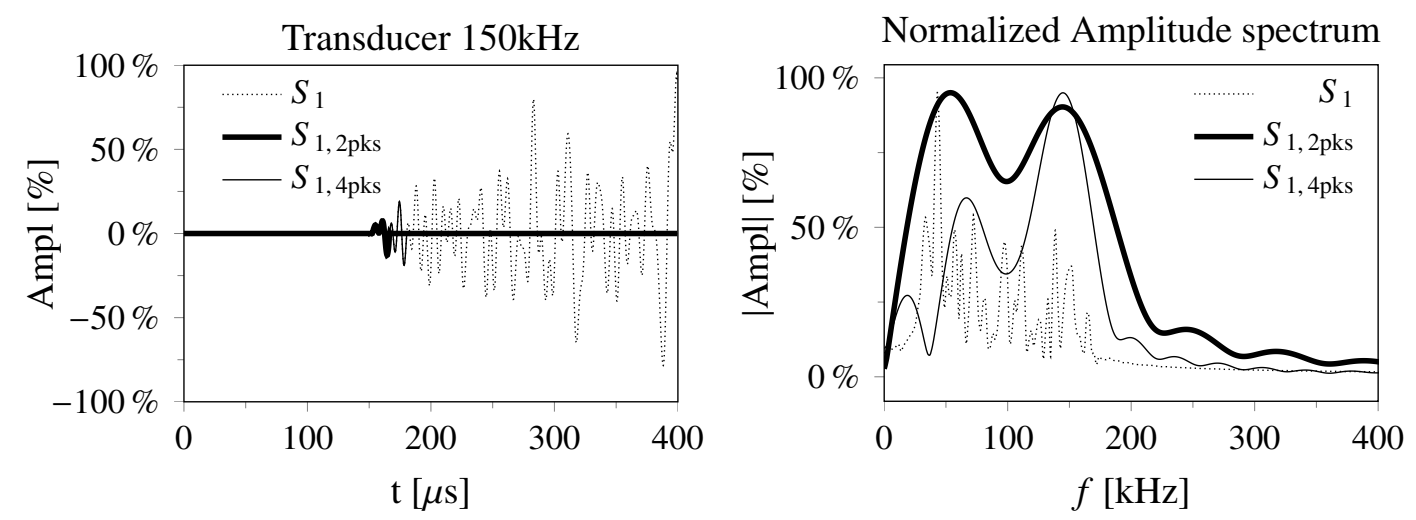

Figure 10: Concrete sample - Normalised amplitude response (Ampl [\%]) and amplitude spectrum (|Ampl|) of signal $S_{1}$ and two portions of it $S_{1,2 \mathrm{pks}}$ and $S_{1,4 \mathrm{pks}}$ recorded at the positions $L_{1}=600 \mathrm{~mm}$, for the transducer with resonant frequency of $150 \mathrm{kHz}$

sample in Fig. 12 and compared with the pulse velocity curve obtained with the Pundit instrument. In both cases, when the whole signal was used in the analysis, an irregular dispersion curve was obtained. This behaviour can be explained as consequence of the limitation of the phase spectrum method in the unwrapping of an irregular phase spectrum. As expected from the frequency spectra in Figs. 6 -10, a smoother representation is obtained when a truncated signal is used, especially with a truncation at the second peak. Even though this last representation provides a slightly better approximation of the expected dispersion curve, it was not taken into consideration for the identification of the length scale parameters of the dynamically consistent model because the windowed signals have been considered not representative of the material behaviour due to the different frequency content included. As consequence, the analysis focused on the pulse velocity obtained with the pulser-receiver instrument and on the velocities of different peaks of the signal. 




Figure 11: Experimental dispersion curves for wave propagation in mortar using the phase spectrum method.

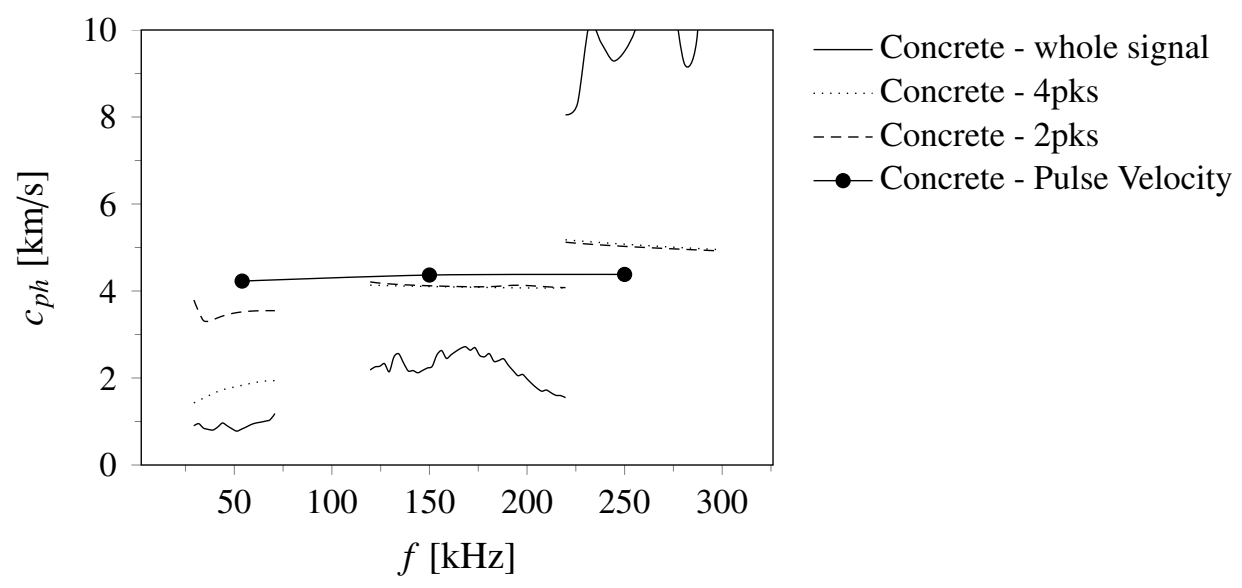

Figure 12: Experimental dispersion curves for wave propagation in concrete using the phase spectrum method.

The pulse velocities for the mortar beam sample are summarised in Table 1, for the three different transducers. In details, the table includes the pulse velocities measured with the Pundit instrument on $L_{1}=195 \mathrm{~mm}, V_{\text {pundit }, S_{1}}$; and $L_{2}=300 \mathrm{~mm}, V_{\text {pundit }, S_{2}}$; the pulse velocities of the first disturbance $V_{p h, 0}$ measured as the ratio between the length difference $L_{2}-L_{1}=105 \mathrm{~mm}$ and the time difference of the first recorded disturbance of the signals $S_{1}$ and $S_{2}$; the velocity of the first peak $V_{1^{s t} P k}$ and second peak $V_{2^{n d} P k}$. It can be observed that in all the cases the pulse velocity slightly increases for increasing resonant frequency of the transducers, with an average increment of about $+300 \mathrm{~m} / \mathrm{s}$. Interestingly, the velocities of the first and second peaks are lower than those recorded by the Pundit, with $V_{2^{n d} P k}$ being the lowest. Analogously, the pulse velocities for the concrete beam sample are summarised in Table 2.

The dispersion curves for the pulse velocities measured using the Pundit instrument on mortar and concrete are compared in Fig. 13. As expected, the velocity for concrete 


\begin{tabular}{c|ccccc}
\hline $\begin{array}{c}\mathrm{f} \\
\mathrm{kHz}\end{array}$ & $\begin{array}{c}V_{\text {pundit }, S_{1}} \\
{[\mathrm{~m} / \mathrm{s}]}\end{array}$ & $\begin{array}{c}V_{\text {pundit }, S_{2}} \\
{[\mathrm{~m} / \mathrm{s}]}\end{array}$ & $\begin{array}{c}V_{\text {pulse }, 0} \\
{[\mathrm{~m} / \mathrm{s}]}\end{array}$ & $\left.\begin{array}{c}V_{1^{s t} P k}[\mathrm{~m} / \mathrm{s}] \\
\hline 54 \mathrm{kHz}\end{array}\right) \begin{array}{c}V_{2^{\text {nd }} P k} \\
{[\mathrm{~m} / \mathrm{s}]}\end{array}$ \\
\hline $150 \mathrm{kHz}$ & 3488 & 3513 & 3281 & 3387 & 3088 \\
$250 \mathrm{kHz}$ & 3721 & 3597 & 3500 & 3387 & 3387 \\
\hline
\end{tabular}

Table 1: Five different evaluation of the pulse velocity $[\mathrm{m} / \mathrm{s}]$ for the mortar beam sample: pulse velocity measured with the Pundit on $L_{1}, V_{\text {pundit, }}$; pulse velocity measured with the Pundit on $L_{2}, V_{\text {pundit, } S_{2}}$; pulse velocity first disturbance $V_{p h, 0}$; pulse velocity first peak $V_{1^{s t} P k}$; pulse velocity second peak $V_{2^{n d} P k}$

\begin{tabular}{|c|c|c|c|c|c|}
\hline $\begin{array}{c}\mathrm{f} \\
\mathrm{kHz}\end{array}$ & $\begin{array}{c}V_{\text {pundit }, S_{1}} \\
{[\mathrm{~m} / \mathrm{s}]}\end{array}$ & $\begin{array}{c}V_{\text {pundit }, S_{2}} \\
{[\mathrm{~m} / \mathrm{s}]}\end{array}$ & $\begin{array}{c}V_{\text {pulse }, 0} \\
{[\mathrm{~m} / \mathrm{s}]}\end{array}$ & $\begin{array}{l}V_{1^{s t} P k} \\
{[\mathrm{~m} / \mathrm{s}]}\end{array}$ & $\begin{array}{l}V_{2^{n d} P k} \\
{[\mathrm{~m} / \mathrm{s}]}\end{array}$ \\
\hline $54 \mathrm{kHz}$ & 4228 & 4191 & 3975 & 4016 & 3997 \\
\hline $150 \mathrm{kHz}$ & 4367 & 4303 & 4280 & 4089 & 4115 \\
\hline $250 \mathrm{kHz}$ & 4381 & 4458 & 4461 & 4449 & 4378 \\
\hline
\end{tabular}

Table 2: Five different evaluation of the pulse velocity $[\mathrm{m} / \mathrm{s}]$ for the concrete beam sample: pulse velocity measured with the Pundit on $L_{1}, V_{\text {pundit, }}$; pulse velocity measured with the Pundit on $L_{2}, V_{\text {pundit } S_{2}}$; pulse velocity first disturbance $V_{p h, 0}$; pulse velocity first peak $V_{1^{s t} P k}$; pulse velocity second peak $V_{2^{n d} P k}$

is higher than the one in mortar, in particular the gap is about $1000 \mathrm{~m} / \mathrm{s}$. This difference is mainly due to the higher stiffness of the concrete if compared with the stiffness of the mortar.

\section{Conclusions}

In the present paper, the experimental ultrasonic wave propagation measurements on concrete and mortar are presented. Narrow band waves of three different frequencies $(54 \mathrm{kHz}, 150 \mathrm{kHz}$ and $250 \mathrm{kHz})$ corresponding to the resonance of the transducers are introduced into the mortar and concrete samples. The pulse velocity provided by the pulse generator has been compared with the velocity of different peaks of the signal. An attempt to evaluate the phase velocity using the phase spectrum method was conducted. The results highlighted how the data obtained using the resonance transducers are not enough accurate to represent the dispersion behaviour in the whole relevant range of frequencies $(0 \mathrm{kHz}-500 \mathrm{kHz})$. The signals recorded with the resonant transducers were not able to provide reliable information outside of their resonance frequency. Considering only the three points available in the dispersion curve 




Figure 13: Dispersion curve for the pulse velocity of the beam made out of mortar and the concrete beam with maximum aggregate size of $12 \mathrm{~mm}$.

for the pulse velocity, an accurate identification of the length scale parameters of the dynamically consistent model was not possible. In order to do that, more accurate experiments using broadband transducers connected to a wave generator, which allows to investigate, for each frequency, the corresponding wave velocity for a more accurate representation of the dispersion curve, are suggested for these analysis. The procedure can then be repeated for different concrete samples made using a specific range of aggregate size and the same cement-aggregate ratio and water-cement ratio. Once these curves will be available, they will be used to identify the length scale parameters and their variation with the different aggregate sizes.

\section{Acknowledgements}

EPSRC (UK) is gratefully acknowledged for funding this research project under grant EP/M004163/1.

\section{References}

1. BS EN 12504-4:2004, . Testing concrete. determination of ultrasonic pulse velocity. 2004.

2. ASTM C215-14, . Standard test method for fundamental transverse, longitudinal, and torsional frequencies of concrete specimens. 2014.

3. Aggelis, D., Polyzos, D.. Wave dispersion phenomena in concrete. In: Advances in Scattering and Biomedical Engineering. World Scientific; 2004, p. 159-165.

4. Popovics, S., Rose, J.L., Popovics, J.. The behaviour of ultrasonic pulses in concrete. Cement and Concrete Research 1990;20(2):259-270. 
5. Aggelis, D., Philippidis, T., Tsinopoulos, S., Polyzos, D.. Wave dispersion in concrete due to microstructure. In: CD-ROM Proceedings of the 2004 Int. Conference on Computational $\mathcal{E}$ Experimental Engineering $\mathcal{E}$ Sciences. 2004, .

6. Malhotra, V.M., Carino, N.J.. Handbook on nondestructive testing of concrete. CRC press; 2004.

7. Jacobs, L.J., Owino, J.O.. Effect of aggregate size on attenuation of rayleigh surface waves in cement-based materials. Journal of engineering mechanics 2000; 126(11):1124-1130.

8. Molero, M., Segura, I., Aparicio, S., Fuente, J.V.. Influence of aggregates and air voids on the ultrasonic velocity and attenuation in cementitious materials. European Journal of Environmental and Civil Engineering 2011;15(4):501-517.

9. Gaydecki, P., Burdekin, F., Damaj, W., John, D.. The propagation and attenuation of medium-frequency ultrasonic waves in concrete: a signal analytical approach. Measurement Science and Technology 1992;3(1):126.

10. Anugonda, P., Wiehn, J.S., Turner, J.A.. Diffusion of ultrasound in concrete. Ultrasonics 2001;39(6):429-435.

11. Becker, J., Jacobs, L.J., Qu, J.. Characterization of cement-based materials using diffuse ultrasound. Journal of engineering mechanics 2003;129(12):1478-1484.

12. Iliopoulos, S.N., Polyzos, D., Aggelis, D.G.. New non-local lattice models for the description of wave dispersion in concrete. In: SPIE Smart Structures and Materials+ Nondestructive Evaluation and Health Monitoring; vol. 9436. 2015, p. 94360C-94360C-10.

13. Carta, G., Brun, M.. A dispersive homogenization model based on lattice approximation for the prediction of wave motion in laminates. Journal of Applied Mechanics 2012;79(2):021019.

14. Mindlin, R.. Micro-structure in linear elasticity. Archive for Rational Mechanics and Analysis 1964;16(1):51-78.

15. Chen, W., Fish, J.. A dispersive model for wave propagation in periodic heterogeneous media based on homogenization with multiple spatial and temporal scales. Transactions-american society of mechanical engineers journal of applied mechanics 2001;68(2):153-161.

16. Askes, H., Bennett, T., Aifantis, E.. A new formulation and $C_{0}$-implementation of dynamically consistent gradient elasticity. International Journal for Numerical Methods in Engineering 2007;72(1):111-126.

17. Bennett, T., Gitman, I., Askes, H.. Elasticity theories with higher-order gradients of inertia and stiffness for the modelling of wave dispersion in laminates. International Journal of Fracture 2007a;148(2):185-193. 
18. Askes, H., Aifantis, E.C.. Gradient elasticity in statics and dynamics: an overview of formulations, length scale identification procedures, finite element implementations and new results. International Journal of Solids and Structures 2011;48(13):19621990.

19. Carta, G., Bennett, T., Askes, H.. Determination of dynamic gradient elasticity length scales. Proceedings of the ICE-Engineering and Computational Mechanics 2012;165(1):41-47.

20. Metrikine, A., Askes, H.. One-dimensional dynamically consistent gradient elasticity models derived from a discrete microstructure. part 1: Generic formulation. European Journal of Mechanics-A/Solids 2002;21(4):555-572.

21. Bennett, T., Gitman, I., Askes, H.. Elasticity theories with higher-order gradients of inertia and stiffness for the modelling of wave dispersion in laminates. International Journal of Fracture 2007b;148(2):185-193.

22. Philippidis, T., Aggelis, D.G.. Experimental study of wave dispersion and attenuation in concrete. Ultrasonics 2005;43(7):584-595.

23. Long, R.. Improvement of ultrasonic apparatus for the routine inspection of concrete. Ph.D. thesis; University of London; 2000.

24. Pialucha, T., Guyott, C., Cawley, P.. Amplitude spectrum method for the measurement of phase velocity. Ultrasonics 1989;27(5):270-279.

25. Papadakis, E.P.. Ultrasonic velocity and attenuation: Measurement methods with scientific and industrial applications. Physical acoustics 1976;12:277-374.

26. Sachse, W., Pao, Y.H.. On the determination of phase and group velocities of dispersive waves in solids. Journal of Applied Physics 1978;49(8):4320-4327. 